\title{
Identità e tolleranza, determinismo morale e religione civile, libero pensiero e teismo, filosofia e superstizione: ineludibili nodi della modernità negli studi recenti di Sutcliffe, Taranto e Champion
}

\section{Giovanni Tarantino}

Storicamente, 1 (2005).

ISSN: 1825-411X. Art. no. 16. DOI: 10.12977/stor521

Adam Sutcliffe, Judaism and Enlightenment, Cambridge, Cambridge University Press, 2003; Justin Champion, Republican Learning: John Toland and the Crisis of Christian Culture, 1696-1722, Manchester-New York, Manchester University Press, 2003; Pascal Taranto, Du déisme à l'athéisme: la libre pensée d'Anthony Collins, Paris, Champion, 2000.

Quando una teoria della tolleranza dell'altro taccia e magari implicitamente irrida quanto divide, e piuttosto insista su quanto accomuna, da ultimo contraddice sé stessa in quanto non conduce all'integrazione e al dialogo, bensì all'assimilazione ad un modello a-storico, all'incomunicabilità, al conflitto. Scrive Chiara Giuntini, introducendo la sua meritoria edizione delle opere del Toland:

Se al di fuori delle cerchie ristrette dei liberi pensatori la religione non potrà mai essere razionale e filosofica, lo sforzo verso la pace religiosa non dovrebbe essere rivolto a uniformare le differenze fra culti e credenze, ma piuttosto a valorizzarne gli aspetti più funzionali al benessere comune e a 
favorire la convivenza di tradizioni diverse, disposte a rispettarsi reciprocamente senza pretendere di imporre a tutti le stesse convinzion[1].

In Judaism and Enlightenment, Adam Sutcliffe, brillante allievo di Jonathan Israel, indaga la straordinaria - e ambigua - influenza dell'ebraismo sugli autori illuministi, rispettosi e avidi studiosi della tradizione rabbinica, propensi a riconoscere in Spinoza - l'ebreo rinnegato - il messia del razionalismo («the Jesus Christ of Reason», p. 140), ma insieme insofferenti del legalismo e del particolarismo ebraici così contrastanti con l'aspirazione laica e universalistica dell'Illuminismo: «Voltaire's persistent hostility towards Judaism in a sense draws into unique focus the problems underlying the general Enlightenment stance towards a minority that appeared profoundly unassimilable to its logic» (p. 233).

Sutcliffe ripropone alcune delle note considerazioni di Adorno e Horkheimer sulla relazione tra idealismo settecentesco e autoritarismo di età fascista. Benché riconosca che «there was clearly no inexorable highway from the tensions of post-Enlightenment modernity to the genocide of Auschwitz and elsewhere» (p. 2) e che «the core values of the Enlightenment - justice, reason, toleration, self-actualisation, freedom of thought and speech provide the fundamental grounds on which the entitlements of minorities such as Jews are protected in modern societies» (p. 11), tuttavia, insistentemente, riconduce il persistente sentimento 'allosemita' nella cultura occidentale (p. 9) alla irriducibilità dell'ebraismo ai canoni illuministici di razionalità, laicità, cosmopolitismo: «The struggles, dilemmas and overarching tragedy of the modern European Jewish experience cannot be understood without an awareness of the Enlightenment paradoxes and contradictions that essentially defined the parameters of the barbed embrace of Jewishness and modernity» (p. 3).

L'interesse rinascimentale per i testi e le lingue dell'antichità creò inedite occasioni di collaborazione tra i classicisti cristiani e gli eruditi ebrei. I 
Riformatori, decisi a ricostruire il testo originale del Vecchio Testamento e a tradurlo in volgare, si volsero diligentemente allo studio della lingua e della letteratura ebraica. In Inghilterra, tra il XVI e il XVII secolo, vi fu una vera fioritura degli studi ebraici: rimediando all'esigua consistenza (dovuta all'espulsione) della comunità ebraica inglese, numerosi studiosi convertiti vi si trasferirono e contribuirono alla formazione di ebraisti indigeni[2]. La pubblicazione nel 1611 della Bibbia di re Giacomo presuppose uno studio accurato del testo greco ed ebraico.

The formation of Protestant national and confessional identities [...] assumed an increasingly overt role in the advance of the discipline. In the decades around 1600 the intellectual commitment to Christian Hebraism was strongest in England and in the Dutch Republic: the two states that were most heavily invested in the formation of new theologico-political identities (p. 26).

L'interesse per la lingua e la letteratura ebraica fu accresciuto anche in ragione di un diffuso millenarismo. Richard Popkin ha accertato come alcuni eventi eccezionali (la Guerra dei Trent'anni, l'invasione turca dell'Europa centrale, la rivoluzione puritana, la predicazione del Vangelo in America e in Africa) parvero a molti il segno dell'imminente ritorno del Messia profetizzato da Daniele. Gli ebraisti cristiani si impegnarono in un programma ambizioso di traduzione dei commentari ebraici col solo intento di anticipare la conversione degli ebrei, ritenuta una pre-condizione necessaria alla seconda venuta del Cristo (p. 166)[3]. Inoltre, la libertà goduta dalla comunità ebraica di Amsterdam e la protezione garantita ad alcuni intellettuali ebrei alla corte parigina di Maria de' Medici consentirono agli ebrei sefarditi di esprimere finalmente «their side of the story»[4]. Eliahu Montalto, il medico personale di Maria de' Medici, poté con sufficiente libertà divulgare violentissimi attacchi al cristianesimo. Johann Cristophe Wagenseil pubblicò nel 1681, in versione latina, il Chizzuk Emunah (sive Munimen Fidei, libello redatto nel $\mathrm{XVI}$ secolo da Isaac Abraham Troki, caraita, per preparare gli ebrei a 
fronteggiare l'offensiva dei missionari cristiani), presentandolo come un caso esemplare dell'orrenda considerazione dei cristiani presso gli ebrei. L'edizione di Wagenseil, intitolata Tela Ignea Satanae e concepita come un armamentario utile alla conversione degli ebrei, comprendeva - e inesorabilmente diffondeva - anche il Nizzahon Vetus, uno dei testi più aggressivamente polemici contro il cristianesimo, redatto probabilmente nel XIII secolo. Devono inoltre ricordarsi la pubblicazione del Tratado da verdade da Ley de Moisés di Saul Levi Morteira e la controversia tra Isaac Orobio de Castro e Phillip van Limborch, riportata dall'ultimo in De Veritatae religionis Christianae: Amica Collatio cum Erudito Judaeo (1687).

Contribuì a una maggiore attenzione alla cultura e alla letteratura ebraiche anche l'interesse per la critica testamentaria maturato in ambienti deisti 0 altrimenti eterodossi. Gli autori che nel XVII e nel XVIII secolo intesero contestare l'attendibilità della rivelazione cristiana, dimostrandone con gli strumenti della critica testamentaria e della filologia l'inconsistenza delle prove, si servirono ampiamente della tradizione esegetica ebraica, ma senza rinunciare a biasimare e a schernire la vacuità e l'irrazionalità dei riti e dei costumi ebraici. Anthony Collins, la cui imponente biblioteca (oltre 10.000 titoli) esemplifica le curiosità intellettuali del suo tempo (pp. 173-5)[5], condivise con altri autori illuministi quell'irrisolta ambiguità tra l'ammirazione per la tradizione culturale ebraica e l'insofferenza per il ritualismo ottuso della massa degli ebrei incolti, sino a dirne, nel Discourse of Free-Thinking, che essi erano «illiterate, barbarous, and ridiculous people»[6].

Nella biblioteca del Collins figuravano numerose storie degli ebrei e grammatiche ebraiche. Dei testi ricordati e descritti da Sutcliffe, il Collins possedeva le Antiquitez judaiques di Jacques Basnage e un'edizione in quattro volumi della Bibliotheca Hebraea di Johann Christoph Wolf, notissimo ebraista di Amburgo e possessore a sua volta di una collezione libraria di 25.000 volumi. E ancora: il De republica Hebraeorum di Carlo Sigonio, il De legibus Hebraeorum ritualibus di John Spencer, la République des Hébreux di Petrus Cunaeus (pp. 40, 43, 44, 81-9). II catalogo d'asta 
riferiva inoltre di una collezione di «Tracts relating to the Jews». E tra i numerosissimi titoli del Toland ricorreva naturalmente il Nazarenus (pp. 197206). Toland aveva condiviso l'apprezzamento di Harrington per l'antica repubblica mosaica e nella prima appendice al Nazarenus auspicò che questa potesse un giorno ricomporsi e prosperare in Palestina (p. 204). Nelle Origines Judaicae aveva inoltre accostato la concezione mosaica della divinità al panteismo spinoziano (p. 199). II «retaggio spinoziano» era altresì evidente nella sottolineatura della «funzione eminentemente politica di Mosè [...] e della preminenza della Legge, dunque dell'aspetto politico-normativo, su quello eminentemente religioso, 'sacro', nella storia del popolo d'Israele, e in tutto il Vecchio Testamento"[7]. Mosè, Gesù e Maometto divengono, nella ricostruzione 'storica' del Toland, i successivi riformatori di una religione unica. II vero cristianesimo non è altro che «reformed Judaism; the true religion being one and the same in substance from the beginning». In Europa erano a lungo circolate numerose redazioni manoscritte del famigerato Traité des trois imposteurs, in latino e in francese, e non tutte riconducibili a un'unica tradizione (pp. 141-143, 186). La precoce ricezione inglese di questi testi mostrò di intendere l'ipotesi storica dell'impostura religiosa come un 'rimedio', piuttosto che una 'diagnosi':

According to the English tradition, the theory of imposture went hand in hand with the theory of the political legislator. Public religions were almost always theologically false, but they could potentially become just instruments for the reform of reason. For the English free-thinkers, theology, if it led to virtue, was benign, but if it led to ignorance, prejudice, and error it was priestly imposture. In England, unlike France, the peculiarly Protestant tradition of the Royal supremacy was adopted by radicals like Stubbe and Toland as an Erastian instrument against corrupting priests[8]

Collins aveva inoltre adunato diversi titoli inerenti alla controversia ebraicocristiana. Possedeva naturalmente il De Veritate religionis Christianae, la Conference between a Papist and a Jew di Richard Mayo (1678), l'Histoire des Juifs 
di Basnage, la Demonstration of the Messias del Kidder, un estratto dal Libro dei Principi di Yosef Albo, intitolato Argumenta quibus nonnullos Fidei Christianae Articulos oppugnant e almeno due edizioni dell'opera di Isaac Troki, una manoscritta, in spagnolo (Fortificacion de la Fé), l'altra in latino ( Munimen fidel) pubblicata dal Wagenseil. Disponeva persino di due manoscritti rarissimi che egli - riferisce Desmaizeaux - aveva acquistato in Olanda a caro prezzo: le Prevenciones Divinas contra la Vana Ydolatria de las Gentes di Orobio de Castro e il Providentia Divina de Dios con Israel del rabbino sefardita Morteira. Di questa ricchissima nonché rara letteratura controversistica il Collins si servì ampiamente nei suoi scritti, deciso a palesare le inconsistenze della dottrina cristiana dimostrando come l'uomo crocifisso sul Golgota non realizzasse l'attesa messianica degli ebrei di un liberatore invincibile e che i miracoli, da soli, non bastassero a provarne la divinità. Bene ha scritto Gerard Cragg: «lf the Bible was a record of Revelation, prophecy and miracle were its twin supports. To these the defenders of orthodoxy made their appeal; against them, therefore, the Deists directed their attack»[9]. Quanto all'uso strumentale e retorico di fonti ebraiche nella letteratura deista è ampiamente condivisibile l'annotazione del Sutcliffe, secondo cui

although Jewish arguments against Christianity were of noteworthy importance in the Early Enlightenment, it is not appropriate to consider them, as some scholars have done, as 'sources' of unbelief. [...] Writers such as Collins and Lévesque de Burigny enthusiastically deployed [Jewish] arguments not because they found them straightforwardly convincing, but because they constituted an extremely intriguing and versatile polemical resource (pp. 174-5).

Pur convenendo con Habermas che «rational thought contains within itself the possibility of overcoming its own limitations» (p. 4), il libro del Sutcliffe ripropone da ultimo il monito - non nuovo, controverso, e tuttavia attualissimo[10] - a guardarsi dai rischi di un 'fondamentalismo della 
ragione':

While the tensions between Judaism and Enlightenment were [...] uniquely intense and historically significant, they are closely related to the more general problematics of the relationship of Enlightenment rationality to whatever it cannot readily encompass. [...] The idea of tolerance can only be defined in opposition to a contrasting notion of intolerance. For Bayle, and also for Spinoza and Locke, Judaism filled the rhetorically indispensable role of philosophical negative, in diametric contrast to which the positive contours of toleration could be delineated. In our own era Islam rather than Judaism is much more frequently cast as the inverse of enlightened toleration, in contrast to which 'western' values are defined and reinforced. [...] The mythic resilience of Judaism holds within it a unique power to call attention to the limits of Enlightenment, and to provide a bulwark against univocal rationalist arrogance and authoritarianism (pp. 6, 225, 261).

E' doveroso domandarsi, come ha scritto Tortarolo su Golem (luglio 2001), se la «religione civile di Rousseau» non sia «diventata religione in-civile e, in qualche caso almeno, teologia politica, [..] identificata con la sacralizzazione più o meno intollerante e aggressiva, intermittente o duratura, sistematica o abborracciata, di quelle 'comunità inventate' per eccellenza che sono la nazione, l'etnia, la razza, la stirpe, la comunità locale»[11]. Purché, tuttavia, dell'llluminismo non si vogliano ricordare i soli 'limiti', né da ultimo ci si intenda sottrarre, «laici e cristiani, italiani, europei e uomini della globalizzazione, alle sfide poste all'universalismo delle differenze (che è una scelta razionale)»[12]. Da alcuni anni, nuovi orientamenti storiografici

sembrano proporsi di sminuire, o trascurare, il lascito delle esperienze eterodosse e laiche, e riproporre la centralità delle istituzioni e delle credenze religiose: «ll filo della continuità riemergente è piuttosto il disciplinamento, l'interazione, il controllo sociale e non la forza incomprimibile dell'eresia come coscienza della coscienza»[13].

Quanto poi alla «concezione 'deistica' dell'eterna accessibilità del vero» o di 
un «intellectual equalitarianism», occorrerà ricordare anche il concorrente e amaro convincimento che alla verità filosofica accedano in pochi e che per gli altri sia opportuno predisporre o preservare una religione civile. Toland più volte indicò in Mosè una figura esemplare di buon legislatore, in quanto «impegnato nella ricerca di un equilibrio fra il rispetto della verità filosofica sulla natura divina e le concessioni alla mentalità di un popolo fin troppo sensibile alle suggestioni del soprannaturale e del miracoloso [...], almeno fino a che non fosse in grado di riflettere e giudicare in modo autonomo». Tuttavia, nel Pantheisticon, «alla fiducia programmatica nella capacità di emancipazione dell'intelligenza dei laici, basata sulla presa di coscienza del diritto universale all'esercizio delle proprie facoltà razionali, si è sostituita una consapevolezza più matura e disincantata degli ostacoli che si oppongono alla diffusione del libero pensiero e della necessità di strumenti alternativi per l'educazione del volgo»[14]. Così il Collins, nella discussione con Clarke sulla libertà dell'uomo, si ritrae infine nel silenzio, quando, paradossalmente, è il suo interlocutore a richiamarlo alla responsabilità sociale di non esporre il volgo a «la bigoterie et la superstition». «Si la liberté est impossible, alors le théisme est exsangue. C'est cela que veut démonstrer Collins; la réfutation complète de Clarke passe par les démonstrations contre la liberté»[15] Ma la libertà - secondo Clarke - è la sola condizione dell'imputabilità morale:

La seule pédagogie qui puisse en détouner les hommes est celle d'une religion qui les tiens pour libres de suivre leur raison. La libre-pensée, sur ce point, est une anti-éducation, parce qu'elle nous apprend que nous ne somme, et ne serons jamais, que le résultat des influences diverses qui s'exercent sur nous. [...] II faut donc que la liberté soit défendue: devoir de théologien, et d'éducateur du genre humain. [...] Le théologien relève le défi de l'incrédulité en vogue à l'époque, n'hésitant pas à en appeler à la responsabilité morale du penseur - de «l'intellectuel» - plutôt qu'à sa foi. [...] La controverse s'arrêta donc sur la victoire de ce dernier [Clarke], victoire 
évidemment convenue, car l'opinion orthodoxe l'emporte toujours sur l'opinion scandaleuse pour des raisons qui n'ont rien de philosophique[16]

La monografia di Pascal Taranto, Du déisme à l'athéisme: la libre pensée d'Anthony Collins, è già diventata un testo di riferimento imprescindibile per gli studi sul Collins. Si fronteggiano ormai due interpretazioni del pensiero collinsiano[17]. La maggior parte dei suoi interpreti, Leland, Stephen, Robertson, Mossner, Gay, O'Higgins, hanno inteso il Collins sicuramente quale un anti-clericale, magari un anti-cristiano, tuttavia un teista sincero, un 'immortalista': dunque, in breve, un deista. Essi hanno mostrato di credere alle prudenti ammissioni di fede dell'autore, pur se contraddette da una lettura complessiva della sua opera. David Berman, invece, e dopo di lui Dybikowski e Taranto hanno piuttosto scrutato gli abili travestimenti del pensiero eterodosso di Collins («the art of theological lying»), occultato ai nemici e agli incolti, ma accessibile a lettori complici e solidali[18] Ai loro occhi Collins non fu davvero un deista, ma al contrario un vero athée de système (p. 18). L'ultimo capitolo del volume di Taranto, laddove ripercorre le interne contraddizioni del teismo, è intitolato «La destruction du théisme» ed egli non esita, nelle conclusioni, a dichiarare «le naufrage de l'apologétique rationaliste» (pp. 424-437). «La réfutation du système théiste de Clarke, archétype de la religion neturelle, signifie la démonstration, sinon de "l'inexistence" de Dieu, du moins de la possibilité de l'atheisme (comme hypothèse mieux fondée). C'est donc sur la faillite du "deisme" de Clarke que notre libre-penseur construit son athéologie» (p. 331; cfr. pp. 357, 399, 400)[19] Numerose asserzioni del Collins paiono espressione, «si l'on veut, d'un panthéisme matérialiste»:

'Si l'on veut', parce que le 'pantheisme' n'est ici un 'théisme' que par la même astuce un peu cynique qui faisait dire à Lysiclès: un 'Dieu' dont on ne connâit aucun des attributs nécessaires pour fonder la religion, n'a de Dieu que le 
nom; lequel peut être conservé pour ne pas se distinguer, et 'c'en est ainsi fait de l'athéisme'. C'est en tout cas la position que Collins assigne à Spinoza: 'Pour autant que je puisse juger des opinions de Straton, de Xénophane, et de quelques autres athées anciens' sans excepter ces modèles de vertu que sont les 'literati' de la Chine, 'pour moi ils tombent d'accord avec Spinoza (lequel dans ses Opera Posthuma a cherché à réduire l'athéisme en système) qu'il n'y a pas d'autre substance dans l'univers que la matière, que Spinoza appelle Dieu, et Straton, la Nature' [Third Answer, p. 386] (p. 332).

Dopo aver lungamente analizzato i fondamenti teorici e i fini del libero pensiero, Taranto si sofferma nel secondo capitolo sugli espedienti narrativi di quanti - e tra essi il Collins -, costretti dai vincoli della censura, dissimularono il loro pensiero, ricorrendo non di rado al ridicolo e all'ironia: «Le 'privilège' de l'ironie, c'est le privilège des plus faibles de se défendre contre les plus fortes» (p. 96). In A Discourse concerning Ridicule and Irony in Writing - che Taranto, contrariamente a O'Higgins, ritiene possa attribuirsi al Collins -, il ricorso all'ironia dissacrante, in quanto presuppone la fallibilità di ogni opinione, è presentato come un antidoto sano alla seriosità dei tanti pretenziosi custodi della verità. II libero pensatore rinuncerà al «privilege of irony» solo quando gli sia garantita libertà di espressione. Inoltre, quanti perseguono l'immoralità del ridicolo e dell'ironia non si avvedono di quanto siano essi stessi ridicoli: «Derrière la rétorsion classique (ceux qui honnissent la satire sont les premiers à en régaler), on trouve un playdoier pour le respect mutuel des opinions dans lequel l'ironie est présentée comme la sanction méritée de l'intolerance» (p. 94). II volume di Taranto procede poi ad un confronto minuzioso tra gli scritti del Collins e le tesi apologetiche confluite nelle Boyle's Lectures, sottolineando oltretutto le implicazioni sociali delle rispettive posizioni filosofiche:

La "négation" pure et simple de "Dieu" n'a guére de sense quand on baigne 
dans une culture théiste. Si l'athéisme de Collins a un sens, ce ne peut être que contre des sistèmes de pensée prévalents, dont il cherche à miner les fondaments. C'est pourquoi nous ne pouvons envisager une recherche de ce genre sans prendre la mesure de la dimension polémique de la libre-pensée. Toute l'œuvre de Collins est une discussion serrée des préjugés qui s'affirment comme des certitudes, quand ils n'ont droit qu'au titre plus modeste d'hypothèses. II faut donc nécessairement savoir à qui il s'oppose pour comprendere à quoi il s'oppose. [...] Pour les théologiens rationalistes, libéraux et/ou newtoniens, l'explication est peut-être plus subtile. Les «mauvais» libres-penseurs étaient perçus comme une menace contre l'ordre «newtonien» en train de se mettre en place. Ils concurrençaient une idéologie puissante et nouvelle, destinée à fournir les cadres conceptuels de l'ordre post-révolutionnaire naissant, héritier d'une tradition anti-radicale, antirépublicaine et anti-puritaine, chrétienne mais, à la différence des orthodoxes, relativement libérale: celle de Boyle, et de ceux qu'on allait appeler «les newtoniens». Au théisme providentialiste de ces derniers, adapté aux aspirations de la nouvelle aristocratie marchande, avec sa glorification du travail, du self-interest, de la free competition, ils opposaient un panthéisme matérialiste qui non seulement ne pouvait pas servir d'alibi au mantien de l'authorité ou de la hiérarchie sociale, mais menaçait encore d'en troubler l'affermissement (pp. 20, 28)[20]

Nella Philosophical Inquiry concerning Human Liberty, Collins ritorna sul tema dibattuto anni prima con Clarke: il concetto di libertà gli appare contraddittorio, in quanto presuppone la possibilità di una causa senza effetto. Se la tradizione cattolica aveva tentato di conciliare la contingenza del futuro con l'onniscienza divina, distinguendo in Dio la prescienza dalla predeterminazione, oppure ipotizzando - i molinisti - un «concorso simultaneo» tra una causa prima (Dio) e una causa seconda (l'agente creato), in Cartesio solo la distinzione tra due sostanze create gli consentiva di concepire l'anima come una realtà del tutto autonoma dal corpo e quindi libera dal determinismo meccanico cui quello è sottoposto[21] Alla dottrina deterministica del Collins è sottesa l'intenzione di minare la presunzione 
clericale di determinare, e giudicare, il giusto e l'ingiusto, l'empio e il savio. Ma le sono anche profondamente connessi il rifiuto del dualismo cartesiano ed una consonanza filosofica con la spinoziana reductio ad unum di pensiero ed estensione. Quanto poi ai contenuti della morale, essi sono determinati dalle esigenze di una pacifica convivenza sociale:

II est le véritable présupposé de tous les défenseurs de la liberté: si elle n'existait pas, il faudrait l'inventer, car elle est le réquisit de la morale et de la religion. [...] C'est à la fin de l'Inquiry que Collins suggère ce que l'on pourrait appeler une morale sans Dieu. Cette morale n'est pas prescriptive, ni même axiologique. Sa forme est celle de l'obligation sociale, qui détermine entièrement son contenu. Récompenses et punition, dit-il, «sont les piliers essentiels de la société», car «l'homme est un agent nécessaire, déterminé par le plaisir et la douleur» Le plaisir ou la douleur qui les accompagnent sont les moteurs essentiels de l'action humaine. Aussi Collins les nomme-t-il bien good) et mal (evil). La vertu consiste donc à faire le bien, c'est-à-dire à accomplir des actions plaisantes, et le vice consiste à accomplir des actions désagréables, lorsque l'on est trompé par les apparences du plaisir. De là, il suit que la seule fin des punitions (et des récompenses) consiste à prévenir le crime, soit en retirant le criminel de la circulation pour l'empêcher de nuire à nouveau, soit en effrayant les hommes pour que la représentation du châtiment, par sa vivacité, contrebalance celle du plaisir apparent des crimes. Car s'il n'y avait pas de châtiment, le plaisir du crime ne serait pas apparent, mais réel. C'est la douleur de la peine qui rend le crime vicieux, ou désagréable, ou immoral. [...] La justice de la punition n'a rien à voir avec un jugement moral porté sur la personne, mais avec le bien de la communauté (sa cohésion). [...] Subjectivement, la morale de Collins est rigoureusement hédoniste: elle n'est que la recherche par chacun de son utile propre, le plaisir. Objectivement, elle se réduit à un conditionnement. Seul le rapport objectif de l'action avec les finalités sociales la rend éventuellement susceptible d'être corrigée. [..] La loi naturelle est du ressort du magistrat (pp. $415,421,422,424)$.

Più che una specifica forma di governo, sono il primato della legge, la 
tolleranza, il perseguimento del bene comune, l'indipendenza delle università e dei giudici e l'autonomia del legislatore dalle ingerenze prelatizie che definiscono il repubblicanesimo di Toland: «[He] was insistent that the word 'commonwealth' did not mean a form of democracy, nor indeed, any particular form of government, 'but an independent community, where the Common Weal or Good of all indifferently is design'd and pursu'd, let the Form be what it will'. [...] It was perfectly possible for government by monarchy to produce the conditions for a 'free state' when regulated and limited»[22]. II volume di Champion, Republican Learning: John Toland and the Crisis of Christian Culture, 1696-1722, analizza il progetto politico tolandiano, evidenziando come le sue idee furono tutt'altro che estranee agli uomini - e alle donne (pp. 53-54, 230, 245) - influenti sugli indirizzi politici e costituzionali britannici. II volume è strutturato in tre parti: la prima descrive il milieu sociale e intellettuale del Toland, «the material and social infrastructure for Toland's 'life of mind'»; la seconda introduce la sua dottrina politica ed il suo impegno nel «public writing»; l'ultima analizza i suoi testi manoscritti e lo descrive quale solerte divulgatore di testi clandestini in elitari circoli europei. Toland collaborò con Trenchard, Moyle, Molesworth e Lord Ashley. Curò le edizioni delle opere di Milton, Ludlow, Sidney e Harrington, un lavoro immane, consentitogli dall'editore John Darby, da molti ritenuto «a true assertor of English liberties» (p. 98). Una considerazione attenta e opportuna dell'allegoria della 'libertà repubblicana' riportata sul frontespizio dell'edizione tolandiana di Oceana (1700) consente a Champion di chiarire esemplarmente in qual modo Toland abbia adattato ai suoi tempi e ai suoi obiettivi i testi repubblicani: Guglielmo III è accostato a Bruto, I'uno e l'altro restauratori della libertà, e sopra di loro stanno i ritratti di Mosè, Solone, Confucio, Licurgo e Numa, alludendo a una continuità con l'antica prudenza di Atene, Roma e Sparta e a certo sincretismo, o «relativismo religioso». Le rappresentazioni in basso di un contesto urbano e di una scena agreste, intitolate rispettivamente 'Commercio' e 'Opificio', identificano nelle transazioni commerciali e nella terra «the 'balanced' premise of the free state» (pp. 106-7) Strenuo sostenitore della successione hannoveriana, 
Toland attinse ai classici repubblicani per contrastare le rivendicazioni degli Stuart e le influenze corruttrici della Chiesa:

All princes were latent tyrants, but this dormant form was habitually only cultivated by the corrupt 'pulpit stuff' of clergymen. The war against tyranny then was fought on the front-line of theological discourses. [...] Since priestcraft made people ignorant, the attack upon it became a key part of a practical political agenda. A consequence of this was that Parliament and nobility, as bearers of reason and virtue, were the best agencies of republican reform. [...] Unlike other neo-Roman theories of free states that were regarded as subversive because they attached a language of liberty to radical forms of representative government, the republicanism of Toland was elite and hierarchical. Its radicalism was found in the fact that it challenged fundamentally the traditional and dominant accounts of political authority that were built on theological foundations. The 'political' problem of tyranny could only be resolved by attention to religious issues. Toleration was the foundation of free citizenship. Toland's republicanism was not radical in either social or institutional ambition. His writings (drawing from Sidney's work) indicate a preference for the rule of an aristocracy of virtue: he did his best to mingle with such people. [..] Although he loudly proclaimed the sovereignty of reason, and indeed of the people, he was more than a simple rationalist, and certainly no democrat (pp. 248-251)[23].

Non soltanto il Toland influì direttamente su uomini di elevata statura politica, redigendo e annotando per loro testi inediti e sovversivi, ma fece altresì ampio uso dei testi consueti dell'apologetica anglicana. La presenza della letteratura clandestina nelle biblioteche di Collins, di Furly, o di Eugenio di Savoia, «the three men who owned the most dangerous collections in Europe» (p. 31), può essere stato un sintomo piuttosto che la causa di una disposizione intellettuale sovversiva. L'intransigenza del Toland non fu tanto determinata dall'accesso privilegiato a un corpus di letteratura eterodossa e clandestina, quanto dall'impulso ad appropriarsi della cultura dominante e a sovvertirne gli esiti e i significati: «Toland, like Furly, Collins, Locke et al. 
were not just interested in reading 'radical' books, but primarily wanted to engage with the arguments of the mainstream. [..] [However] his purpose was to engage, not with the details of any specific theological doctrine, but with a more fundamental discussion of the politics of knowledge» (pp. 41, 79)

Champion conclude il suo studio ribadendo l'originalità e la specificità del pensiero di Toland (e la plausibilità di un «English Enlightenment») e contestando a Jonathan Israel che tutto debba ricondursi a Spinoza:

Where Spinoza deployed his rabbinical learning in a single-minded account of the status of Old Testament scripture, Toland drew on a much wider range of erudition. Where Spinoza condensed republican political theory into a few chapters of commentary on the contemporary writing of people like Peter de la Court, Toland produced a massive canon of commonwealth writings, carefully made bespoke for contemporary purposes. Where Spinoza wrote in Latin and his posthumous ideas were circulated clandestinely, Toland used a full range of vernacular styles for both public and private polemic (p. 253)

II 'commonwealth party' fu sconfitto dal pragmatismo di Robert Walpole che preferì avvalersi della Chiesa anglicana per consolidare la sua egemonia politica. Tuttavia, Toland - e con lui altri liberi pensatori, apparentemente isolati e ininfluenti - lasciò un archivio ingente di testi e idee che l'llluminismo più tardo e maturo («the 'High' Enlightenment») avrebbe utilmente impiegato nel suo attacco all'irrazionalismo e alla superstizione. «We still live in a modernity shaped by this afterlife» (p. 254)[24]

\section{Note}

[1] C. Giuntini, Introduzione a Opere di John Toland, Torino, UTET, 2002, 773: 63.

[2] Vedi H. J. Schoeps, Barocke Juden, Christen, Judenchristen, Bern- 
München, Francke, 1965; R. M. Healey, The Jew in Seventeenth-Century Protestant Thought, "Church History», 46 (1977), 63-79; D. S. Katz, Philo-semitism and the Readmission of the Jews in England 1603-1655, Oxford, Oxford University Press, 1982; J.-F. Gilmont, La Réforme et le livre: l'Europe de l'imprimé 1517-v.1570, Paris, Cerf, 1990

[3] Vedi R. H. Popkin, Some Aspects of Jewish-Christian Theological Interchanges in Holland and England 1640-1700, in J. van den Berg, E. van der Wall (eds), Jewish Christian Relations in the Seventeenth Century, London, Kluwer Academic Publishers, 1988; Id. (ed.), Millenarianism and Messianism in English Literature and Thought, Leiden, Brill, 1988.

[4] R. H. Popkin, Jewish Anti-Christian Arguments as a Source of Irreligion from the Seventeenth to the Early Nineteenth-Century, in Atheism from the Reformation to the Enlightenment, ed. by Michael Hunter and David Wootton, Oxford, Clarendon Press, 1992, 161-162.

[5] Cfr. G Tarantino, Lo scrittoio di Anthony Collins (1676-1729): 1. I libri e i tempi di un libero pensatore; 2. Edizione del catalogo manoscritto della biblioteca collinsiana (1720), tesi di dottorato, Università degli Studi di Firenze, 2004.

[6] [A. Collins], A Discourse of Free-Thinking, Occasion'd by the Rise and Growth of a Sect Call'd Free-Thinkers, London, 1713 (1st edn), 157.

[7] P. Bernardini, Introduzione a John Toland, Ragioni per naturalizzare gli ebrei in Gran Bretagna e Irlanda (1714), Firenze, Giuntina, 1998, p. 71. Sul paradigma mosaico come alternativo a quello machiavelliano nelle teorie repubblicane della prima età moderna si vedano inoltre E. H. Kossman, Dutch Republicanism, in L'età dei Lumi. Studi storici sul Settecento europeo in onore di Franco Venturi, 2 voll. Napoli, I, 453-486; L. Campos Boralevi, Per una storia della Respublica Hebraeorum come modello politico, in V. I. Comparato, E. Pii (eds.), Dalle 'Repubbliche' elzeviriane alle ideologie del '900, Firenze, 1997, 17-33; Ead., Classical Foundational Myths of European Republicanism: The Jewish Commonwealth 
, in Republicanism: A Shared European Heritage, ed. by M. van Gelderen and Q Skinner, 2 voll., Cambridge, Cambridge University Press, 2002, I, 247261.

[8] J Champion, Legislators, Impostors, and the Politic Origins of Religion: English Theories of 'Imposture' from Stubbe to Toland, in S Berti, F. CharlesDaubert, R H. Popkin (eds.), Heterodoxy, Spinozism, and Free Thought in Early-Eighteenth-Century Europe: Studies on the Traité des Trois Imposteurs , Dordrecht-Boston-London, Kluwer Academic Publishers, 1996, 333-356: 356. Cfr. Id., Republican Learning cit., 173 ss.; S. Suppa, L'ebraismo nell' Encyclopédie» di Diderot e D'Alembert. Ovvero: regole, Scrittura e memoria, in un ordine senza Stato, «II Pensiero Politico», XXXV (2002) [Politeia biblica, a cura di L. Campos Boralevi e D. Quaglioni], 464480: 477.

[9] G Gragg, Reason and Authority in the Eighteenth Century, Cambridge, Cambridge University Press, 1964, 84.

[10] Si pensi soltanto alla sconcertante omelia contro la «dittatura del relativismo, che non riconosce nulla come definitivo e che lascia come ultima misura soltanto il proprio io e le sue voglie», pronunciata durante la messa 'Pro eligendo Romano Pontifice' da Joseph Ratzinger e che tanto ha contribuito alla sua elezione papale Cfr. M. L. Salvadori, La nuova alleanza tra fede e ragione, «La Repubblica», 10 settembre 2005, 15 e H. W. Blom, Secularization in Dutch Political Theory: Lambert Van Velthuysen (16221685), in La formazione storica dell'alterità. Studi di storia della tolleranza nell'età moderna offerti a Antonio Rotondò, promossi da H. Méchoulan, R. H. Popkin. G. Ricuperati, L. Simonutti, Firenze, Olschki, 2001, 3 voll., II, 559575: 559. 
[11] Cfr. S. Forti, Totalitarismo e religioni politiche, in G. Paganini, E. Tortarolo (a cura di), Pluralismo e religione civile: una prospettiva storica e filosofica, Atti del Convegno di Vercelli (Università del Piemonte orientale), 24-25 giugno 2001, Milano, Mondadori, 2004, 213-229: 228.

[12] G. Ricuperati, Tolleranza come problema storiografico della modernità, «l castelli di Yale. Quaderni di filosofia», 7 (2004), 31-47: 47.

[13] Ibid., p. 39. Cfr. C. Borghero, I/ giuramento dell'infedele: stato di diritto, tolleranza e obbligazione politica, ibid., 9-29: 10: «Da qualche tempo stanno prendendo piede versioni conciliatrici, come se la tolleranza fosse scaturita da una sorta di autolimitazione, conseguenza di uno spontaneo riconoscimento dei diritti degli altri, e non sia stata invece il risultato drammatico di un processo di limitazione dell'ingerenza delle chiese nella sfera pubblica o, per dirla con linguaggio hegeliano, il risultato di una lunga lotta del rischiaramento contro il fanatismo e la superstizione» $\mathrm{Si}$ considerino, per la Gran Bretagna, gli opposti orientamenti storiografici documentati in A. Houston \& S. Pincus (eds.), A Nation Transformed. England After the Restoration (Cambridge-New York, Cambridge University Press, 2001), J. C. D. Clark, English Society 1660-1832. Religion, Ideology and Politics during the Ancient Regime (Cambridge, Cambridge University Press, 2000) e W. Gibson \& R. G. Ingram (eds.), Religious Identities in Britain, 1660-1832 (London, Ashgate, 2005). O, ancora, si confrontino D. M. McMahon, Enemies of the Enlightenment. The French CounterEnlightenment and the Making of Modernity, New York, Oxford University Press, 2001 e S. J. Barnett, The Enlightenment and Religion. The Myths of Modernity, Manchester, Manchester University Press, 2003.

[14] Giuntini, Introduzione a Opere di John Toland cit., 54-56, 65, 71. 
[15] Taranto, Du déisme à l'athéisme cit., 344. Cfr. I. Rivers, Reason, Grace, and Sentiment, Cambridge, Cambridge University Press, 2 voll, II, 2000, 6465; J. Harris, Of Liberty and Necessity: The Free Will Debate in EighteenthCentury British Philosophy, Oxford, Clarendon Press, 2005, 62, 63.

[16] Ibid., 416-417. Cfr. Borghero, Il giuramento dell'infedele cit., 25-26.

[17] Cfr. A Thomson, Le Discourse of Free-Thinking d'Anthony Collins et sa traduction française, «La Lettre clandestine», 9 (2000), 95-116: 95.

[18] Vedi D. Berman, Deism, Immortality, and the Art of Theological Lying, in Deism, Masonry, and the Enlightenment: Essays honoring Alfred Owen Aldridge, ed. by J. A. Leo Lemay, Newark: University of Delaware Press/London and Toronto: Associated University Presses, 1987, pp. 61-78: 61 Cfr. Perez Zagorin, Ways of Lying: Dissimulation, Persecution, and Conformity in Early Modern Europe, Cambridge MA/London, Harvard University Press, 1990, 10-11

[19] Cfr. G. Mori, Revue de Pascal Taranto, Du déisme à l'athéisme: la libre pensée d'Anthony Collins (Paris: Champion, 2000), «La Lettre clandestine», 9 (2000), 394-395: «Pour ce qui concerne la pensée philosophique de Collins, il s'agit d'une pensée anti-dogmatique, qui se fonde sur une attaque résolue contre les principes de la méthaphysique chrétienne. Collins n'offre pas une 'preuve de l'inexistence de Dieu', sinon sous forme 'apagogique', c'est-à-dire en se fondant 'sur la faillite du théisme' chrétien (p. 267). L' 'athéologie' de Collins (p. 331) est donc surtout une anti-théologie, qui n'est pas très éloignée en tant que telle de celle de Pierre Bayle, dont Collins suit la démarche de manière constante et indéniable. En effet, l'athéisme naît selon Collins à partir 'des propes présupposés du théisme' (p. 432) - ce qui constitue la conclusion même de l'itinéraire philosophique baylien. [...] $\mathrm{Si}$ Collins fut un 'stratonicien moderne' ( $p .381$ ), ce fut aussi grâce à sa lecture très attentive du Dictionnaire et de la Continuation des pensées diverses».

[20] Cfr. J. I. Israel, Radical Enlightenment. Philosophy and the Making of Modernity 1650-1750 
, Oxford University Press, 2001, 11-12.

[21] Cfr. M. Mori, Libertà, necessità, determinismo, Bologna, II Mulino, 2001, 16, 17; Harris, Of Liberty and Necessity cit., 10-11.

[22] Champion, Republican Learning cit., 123, 124; cfr. ibid., p. 110.

[23] "Toleration was the source of 'science', persecution ('the root of ignorance') produced 'sedition and troubles'. Establishing an entire liberty was then not only virtuous, but politically expedient too 'as it furnishes the King with more hearts, and the nation with more hands", ibid., 147; cfr. 1278, 144.

[24] «Far from withering away into the foothills of the 'Enlightenment', theological culture and authority retained a persistent social power into the nineteenth century. While reinforcing the point of how deeply entrenched theological perspectives on the world were in the early eighteenth century, this work will also explore how it was possible to unpick some of the power of these discourses and institutions. To acknowledge the persistence of theological imperatives is not the same as claiming it was either unchangeable or uncontested», ibid., 6 . 